\title{
Über die direkte Konstruktion des perspektiven Umrisses von allgemeinen Schraubflächen.
}

\author{
Von Franz Palm in Wien.
}

Um den perspektiven Umriß einer allgemeinen Schraubläche zu ermitteln, wurden bisher immer die Zentralrisse einer Reihe von Schnitten, z. B. der Normalschnitte, konstruiert und deren Einhüllende gezeichnet. In dieser Arbeit soll eine direkie Konstruktion des perspektiven Umrisses gegeben werden. Im ersten Teil der Abhandlang wird die Konstruktion für beliebige Schraubflächen entwickelt, im zweiten Teil derselben werden besondere Schraubflächen behandelt, die eine einfachere Konturbestimmung gestatten.

\section{I.}

Wir denken uns die Schraubfläche $\Phi$, deren perspektiver Umriß konstruiert werden soll, durch die Normalkurve $N^{1}$ ) und die Schraubung gegeben. Die Sehraubachse $A$ sei vertikal. $\mathrm{Ob}$ wir dieselbe in der Bildebene annehmen oder nicht, ist für die Konstruktion gleichgiltig. Es erweist sich als zweckinäpig, die Grundebene der Perspektive mit der Horizontebene zusammenfallen zu lassen. Sei also (Fig. 1) $\Pi_{2}=\Pi$ die Bildebene, $\Pi_{1}$ die Horizontebene, a das Projektionszentrum, $H$ der Horizont, $h$ der Hauptpunkt. Zur Festlegung der Schraubung sei die Bahnschraublinie $S$ eines Punktes der Normalkurve $N$ gezeichnet. ${ }^{2}$ )

Der Grundgedanke der Konstruktion ist der, die a u f in er Bahnschrablinie liegenden Punkte des wahren Umrisses zu ermitteln.

Dazu gehen wir von der folgenden Abbildung aus:

Der Tangente $T_{S}$ an die Bahnschraublinie $S$ in deren in der Normalebene $\Pi_{p}$ der Schraubung liegenden Punkte $p$ ordnen wir ihren Fluchtpunkt $\bar{p}$ füs $\Pi_{p}$ als Bildebene und $o$ als Projektionszentrum zu. Dieser Punkt $\bar{p}$ soll einfach der der Tangente $T_{S}$ zugeordnete Punkt heiben. Durch diese Abbildung erscheint

1) Wegen der Bezeichnungsweise vgl. man E. Müller, Lehrbuch der darstellenden Geometrie für Technische Hochschulen, Leipzig, Teubner 1908, I. Bd., p. 7-11.

2) Diese Schraublinie $S$ wird bei dex späteren Konstruktion verwendet. 
jeder Tangente von $S$ ein bestimmter Punkt zugeordnet. Der Abwickelbaren längs $S$ ist eine Raumkurve $\bar{S}$ zugeordnet, die sich auf dem durch $o$ gelegten Richtkegel $\Phi_{1}$ dieser Abwickelbaren befindet. Der Charakter des Grundrisses dieser zugeordneten Kurve $\bar{S}$ ist leicht zu erkennen. Sei $L$ die Parallele durch $o$ zum Grundrib der Tangente an $S$ in deren Sehnittpunkte $p_{o}$ mit der Horizontebene. Wenn wir um den Winkel $\varphi$

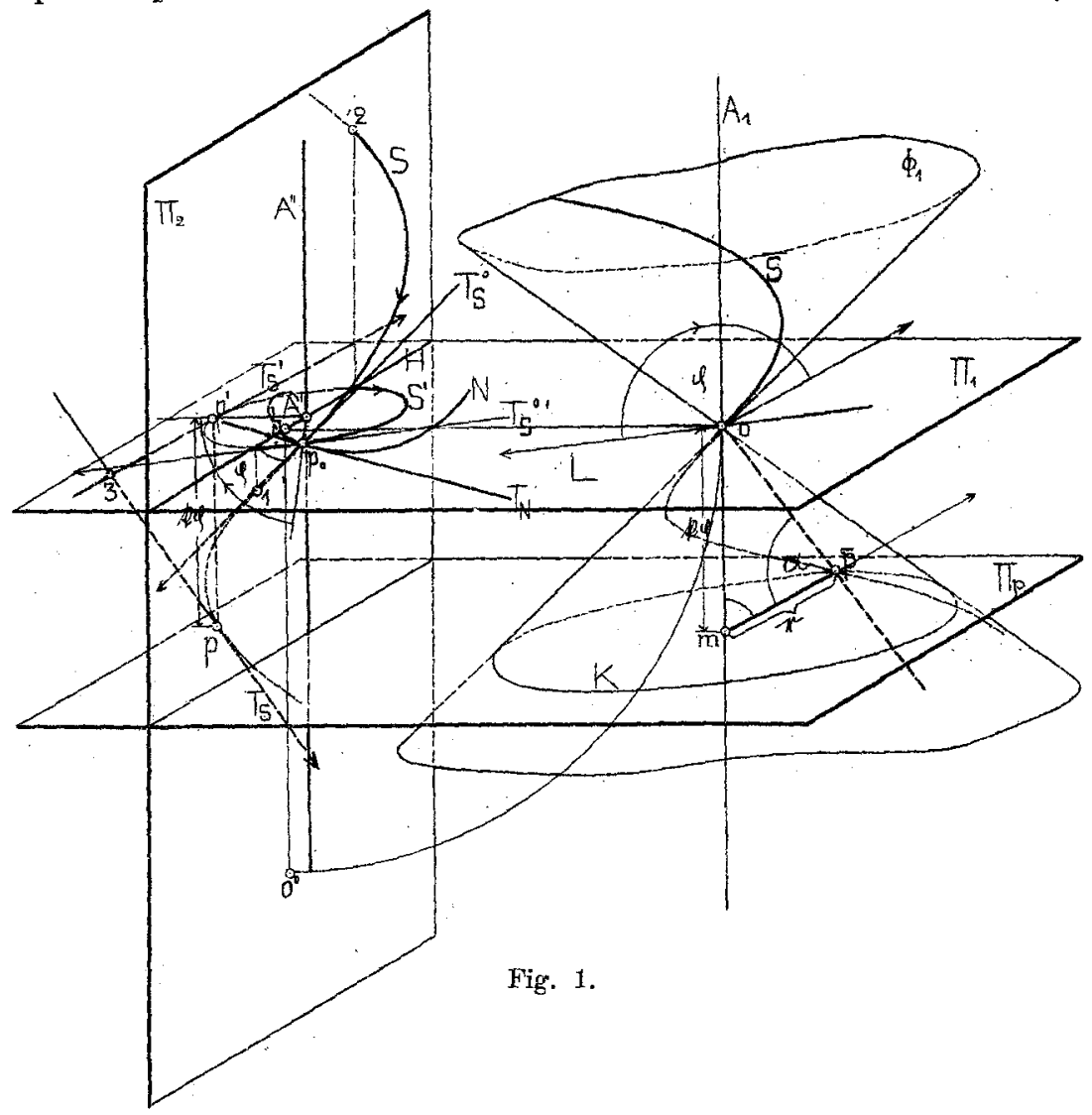

verschrauben, gelange $p_{0}$ nach $p$. Dem entspricht eine Drehung von $L$ um denselben Winkel. Gleichzeitig entfernt sich der Grundriß $\overline{p^{\prime}}$ des der Tangente an die Bahnschraublinie $S$ zugeordneten Punktes $\bar{p}$ von dem Punkte $o$, der $T_{S_{q}}$ entspricht, um die Streeke $r=1 \varphi \cot \alpha$, wenn $\alpha$ die Neigung der Schraublinie $S$ gegen $\Pi_{1}$ bedeutet. Die Polargleichung von $\overline{S^{\prime}}$ für $o$ als Pol und $L$ als Achse ist mithin $\mathfrak{r}=\mathfrak{l} \varphi \cot \alpha$, wobei $\mathfrak{l} \cot \alpha$ konstant ist; daher:

Der Grundrib der zugeordneten Kurve $\bar{S}$ ist eine Archimedische Spirale, deren Pol das Projek- 
tionszentrum 0 ist und deren Achse zum GrundriB der Tangente an die Bahnschraublinie $S$ in ihrem Sehnittpunkte mit der Horizontebene parallel ist. ${ }^{3}{ }^{4}$ )

Wir fragen nun, für welche Lage der Normalkurve $N$ der Punkt $p$ ein Punkt des wahren Umrisses wird. Wenn wir die Tangentialebenen aus 0 an die Schraubfläche $\Phi$ stets durch die Tangente $T_{S}$ an die Bahnschraublinie $S$ und die Tangente $T_{N}$ an die Normalkarve $N$ festlegen, gilt:

In den Punkten des wahren Umrisses àer Schraubläche $\Phi$ enthält die Tangente an die Normalkurve den der Tangente an die Bahnsehraublinie zugeordneten Punkt.

Den Tangenten in den auf einer Bahnschraublinie $S$ liegenden Punkten des wahren Umrisses sind die Schnittpunkte der zugeordneten Kurve $\bar{S}$ mit der geraden Regelschraubfläche $\Psi=\left(T_{N}, A, \mathfrak{f}\right)$ zugeordnet.

Hieraus ergibt sich folgende Konstruktion: Man konstruiere die Archimedische Spirale $\overline{S^{\prime}}$; verzeichne die Normalkurve $N$, die 'Tangente $T_{N}$ im Punkte $p$ an $N$ und den Radius $A^{\prime} p$ von $S^{\prime}$ auf einem $\mathrm{P}$ auspapier, befestige dasselbe in $A^{\prime}$ mit einem Reißnagel und drehe es um $A^{\prime}$. Gleichzeitig lege man ein Dreieck stets so in die Zeichenebene, dafo eine Kante durch $o^{0}$ geht und zu $A^{\prime} p$ senkrecht steht. Treffen sich bei der Bewegung diese Dreieckseite and $T_{N}$ in einem Punkte $\overline{a^{\prime}}$ von $\overline{S^{\prime \prime}}$ so drücke man diesen Punkt $\overline{a^{\prime}}$ und den zugehörigen Punkt $a^{\prime}$ mit einem Pausstift durch.

Auf diese Weise ergeben sich in Fig. 2 die drei auf der Bahnschraublinie $P$ liegenden Punkte $a, b, c$ des wahren Umrisses im Grundrif. Der Aufrif des Punktes $a$ wird erhalten, indem man die der Schraubung um den Winkel $\varphi=\Varangle p A^{\prime} a^{\prime}$ entsprechende Höhe z sucht. Zur Ausführung dieser Konstruktion ${ }^{5}$ ) mulb eine Bahnschraublinie gezeichnet vorliegen. Wir verwenden hiezu die zur Festlegung der Schraubung $(A, \mathfrak{l})$ gegebene Schraublinie $R$. In Fig. 2 ist die Konstruktion von $a^{\prime \prime}$ vollständig" angegeben.

Hat man nach dieser Methode den wahren Umriß $U$ konstruiert, so kann man in bekannter Weise die charakteristischen Punkte der Kontur, z. B. die Spitzen, genau angeben.

${ }^{3}$ ) In anderer Fassung ist hierin ein lang bekanntes Resultat zu erkennen. Betrachten wir $L$ als Erzengende einer Wendelfäche $\Phi_{2}$ mit der Achse $A_{1} \| A$ und demselben Parameter 1 wie die gegebene Schraubläche $\Phi, \bar{S}$ ist ersichtlich der Schnitt der Wendelfläche $\Phi_{2}$ mit dem Drehkegel $\Phi_{1}$. Daß der Normalriß dieser Schnittkurve auf eine zu $A_{1}$ normale Ebene eine Archimedische Spirale ist, war schon Pappus bekannt. Vgl. G, Loria, Spezielle algebraische und transzendente Kurven, Leipzig, Teubner 1902, pag. 402.

4) Die Archimedische Spirale $\overline{S^{\prime \prime}}$ ist mit $S^{\prime}$ gleich orientiert.

5) Vgl. E. Müller, Lehrbuch d. darst. Geom. I. pag. 333 und dessen Vorlesungen über Schraubflächen an dex Technischen Hochschule in Wien. 


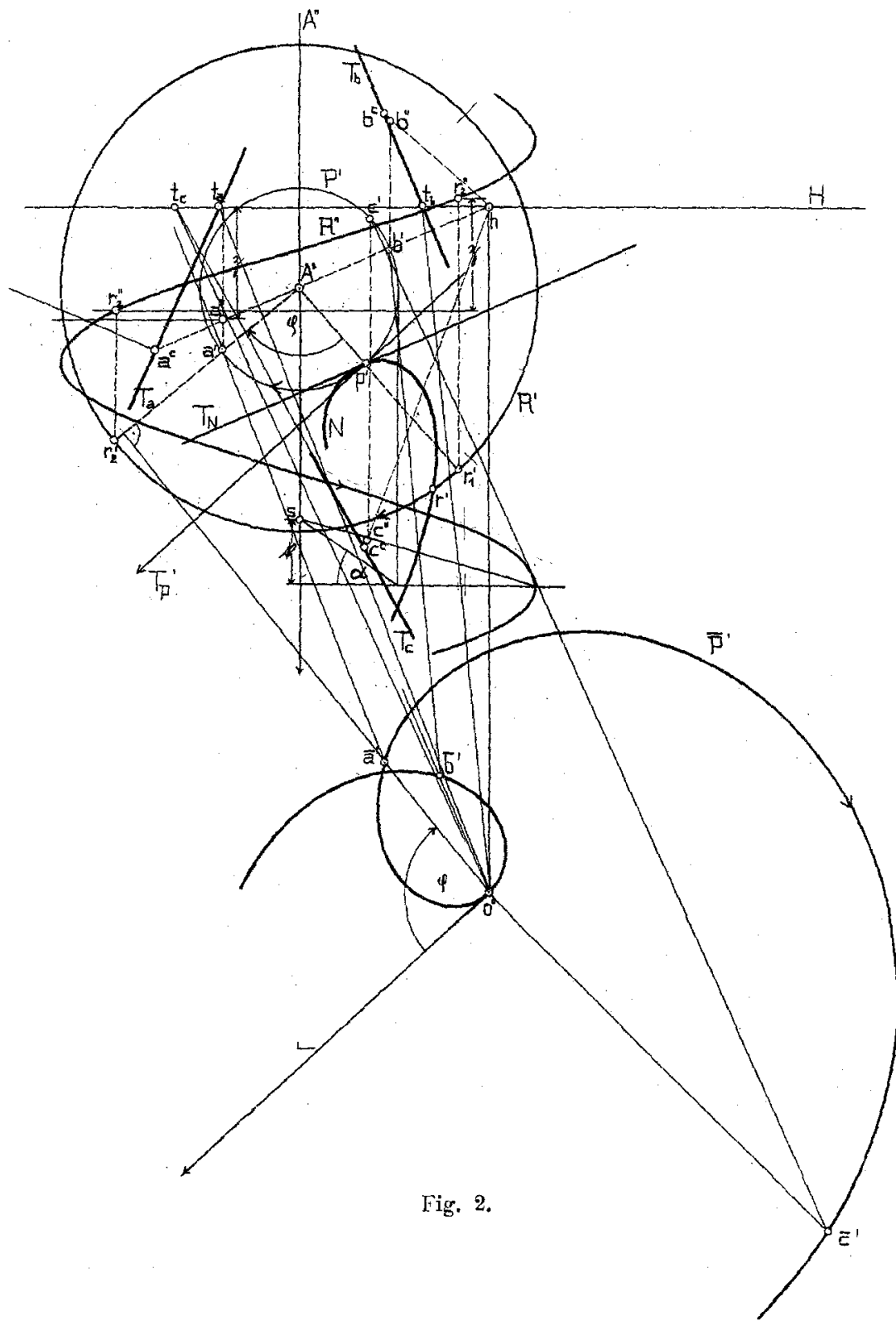

Konstruktion der Konturtangente. Die Konturtangente im Punkte $a^{n}$, der sich in bekannter Weise aus $a^{r}$ und $a^{\prime \prime}$ ergibt, ist die Schnittlinie der Tangentialebene $\tau$ in $a$ mit der Bildebene. Am einfachsten gestaltet sich die Konstruktion, wenn 
man den Schnittpunkt der Tangentialebene $\tau$ mit dem Horizont $H$ ermittelt. Die Spur der Tangentialebene $\tau$ in der Normalebene durch $a$ ist $a \bar{a}$, ihre Spur in der Horizontebene geht durch $o$ parallel zu $a \bar{a}$.

Der Schnittpunkt $t_{a}$ dieser Parallelen $o^{0} t_{a}$ mit dem Horizont $H$ ist schon ein Punkt der Konturtangente in $a^{c}$. Für sehr nahe dem Horizont gelegene Konturpunkte wird man der Genauigkeit der Konstruktion wegen einen entfernteren Punkt der Spur von $\tau$ in $\Pi$ verwenden, was die Konstruktion ein wenig modifiziert.

Der Fall, dab die Schraubachse $A$ zur Vertikalebene der Perspektive parallel, jedoch gegen die Bildebene geneigt ist, läbt sich auf den betrachteten zuruickfübren. ${ }^{6}$ )

II.

Die Flächen, die durch Schraubung einer Schraublinie $S \mathrm{um}$ eine zu ihrer Achse $A$ parallele Schraubachse $G$ entstehen, gestatten eine weitaus einfachere und $\nabla 011 \mathrm{kommen}$ strenge Konturbestimmung in Perspektive, wenn man die Resultate, zu denen Herr Prof. E. Müller ${ }^{7}$ ) gelangte, verwertet. E. Müller hat zuerst den Charakter dieser Flächen als Schiebflächen erkannt und zugleich gezeigt, 'daß̧ es die einzigen Schraubfächen sind, die auch durch Schiebung einer starren Kurve erzeugt werden können. Die folgende Konstruktion des perspektiven Umrisses dieser Schraubflächen stützt sich auf die für Schiebflächen charakteristische Eigenschaft, daß die Tangentialebenen in den Punkten einer erzeugenden Kurve $S$ einen $\mathrm{Zylinder}$ umbüillen.

Wir betrachten vorerst einen Sonderfall, die zyklische Schraubfläche, d. h. diejenige Fläcbe, die durch Schraubung eines starren Kreises $N$ um eine zu seiner Ebene senkrechte Achse $A$ entsteht. Diese Fläche ist eine Schiebfläche, von der die Erzeugenden der zweiten Schar mit der Bahnkurve $M$ des Mittelpunktes $m$ von $N$ kongruent und gleichgestellt sind. Die Tangentialebenen in den Punkten von $N$ umhüllen einen Zylinder. Der Eluchtpunkt der Zylindererzeugenden für die Ebene von $N$ als Bildebene und $o$ als Projektionszentrum beschreibt bei der Schraubung $(A, \hat{)})$ die der Bahnkurve $M$ des Mittelpunktes $m$ von $N$ zugeordnete Kurve $\widetilde{M}$. Die Konturbestimmung wird daher in der folgenden Weise durchzuführen sein: Man konstruiere die Archimedische Spirale $\overrightarrow{M^{\prime}}$. (Fig. 3) Einer Drehung der Normalkurve $N^{\prime}$ um $A^{\prime}$ um den Winkel $\varphi$ entspricht eine Drehung von $L$ um denselben Winkel, wodurch der zugeordnete Punkt $\overline{p^{\prime}}$ gegeben

$\left.{ }^{6}\right)$ Vgl. Niemtschik, Direkte Konstruktionen der Konturen von Rotationsflächen in orthogonalen und perspektivisehen Darstellungen, Sitzungsber. Akad. Wien Bd 52, II. Abtg. (1865) p. 605,

7) E. Muiller: Über Schiebtächen, deren eine Erzeugendenschar aus gew,öhnlichen Schraublinien besteht, Sitzungsber. Alad. Wien, Bd. 118, Abtg. II a, (1909) pag. $3-13$. 


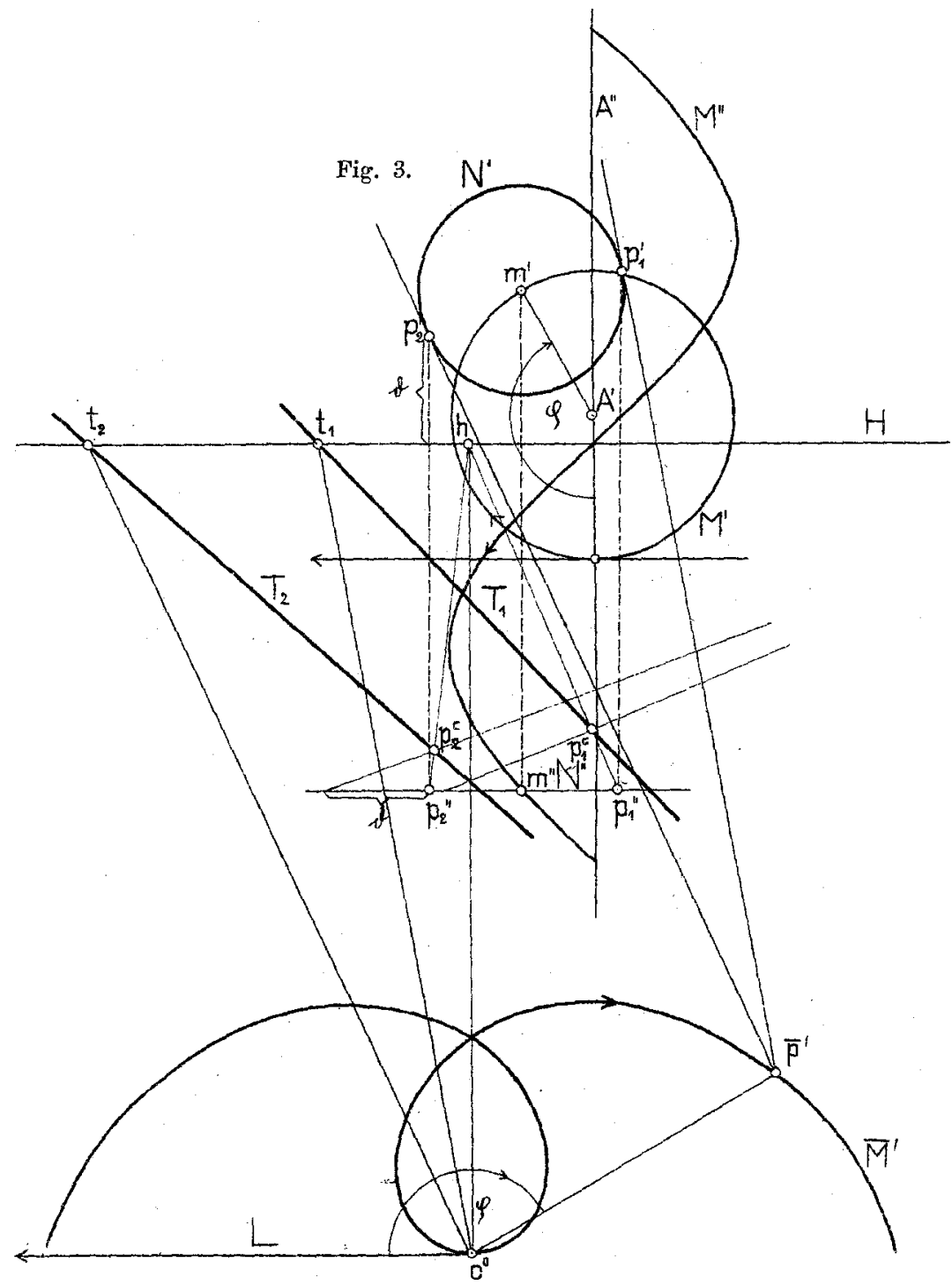

ist. Die Berührungspunkte $p_{1}{ }^{\prime}, p_{2}{ }^{\prime}$ ans $\overline{p^{\prime}}$ an $N^{\prime}$ sind bereits Punkte des wahren Umrisses im Grundriß. Die Aufrisse $p_{1}{ }^{\prime \prime}, p_{2}$ " liegen auf der Geraden $N^{\prime \prime}$. Die Konturtangenten $T_{1}, T_{2}$ werden in derselben Weise ermittelt wie in Fig. 2.

Wir betrachten nun den allgemeinen Fall. Die Fläche, die durch Schraubung einer Schraublinie $S$ mit der Achse $A$ um eine za $A$ parallele Gerade $G$ entsteht, ist eine Schiebfläche, von der die eine Erzeugendenschar aus den bei der Schraubung er- 
haltenen Lagen von $S$, die andere Erzeugendenschar aus Schraublinien $\bar{S}$ mit zu $A$ parallelen Achsen besteht, die den Parameter $\mathfrak{f}_{1}-\mathfrak{l}$ und den Halbmesser $\overline{G^{\prime} A^{\prime}}$ haben, wenn $\mathfrak{l}_{1}$ den Parameter der Schraubung and $\mathfrak{t}$ den Parameter der Schraublinie $S$ bedeutet. Wir betrachten $\bar{S}$ als erzengende Kurve und $S$ als Richtkurve der krummen Schiebung. Die Tangentialebenen in den Punkten der Schraublinie $\bar{S}$ an die Fläche umhüllen einen Zylinder, ${ }^{8}$ ), dessen Erzeugenden parallel sind zur Richtung jener infinitesimalen Schiebung, die $\bar{S}$ in die Nachbarlage überführt, und die durch die Tangente im Schnittpunkte $p$ von $\bar{S}$ and $S$ an $S$ gegeben ist. $\mathrm{Um}$ an diesen Zylinder die T'angentialebenen aus $o$ zu legen, bestimmen wir dessen Schnittkurve $\bar{S}^{s}$ mit der Horizontebene. $\overline{S^{s}}$ ist der Schrägrif von $\bar{S}$ für die Richtung der infinitesimalen Schiebung, die $\bar{S}$ in die Nachbarlage überführt, als Projektionsstrahlenrichtung.

In Fig. 4 ist die durch den Schnittpunkt $p$ von $S$ mit der Horizontebene gehende Schraublinie $\bar{S}$ der andern Schar und der Schrägrib $\overline{S^{s}}$ von $\bar{S}$ für die Tangente $L$ in $p$ an $S$ als Projektionsstrahlenrichtung konstruiert. An diesen Schrägriß $\overline{S^{s}}$ ziehen wir aus $0^{0}$ die möglichen Tangenten ${ }^{9}$ ) und erhalten auf den Parallelen zu $L$ durch die Berührungspunkte die auf $\bar{S}$ liegenden Punkte des wahren Umrisses. Der Grundrib $a^{\prime}$ ergibt sich aut $\bar{S}^{\prime}$; indem wir durch $a^{s}$ die Parallele zu $L^{\prime}$ ziehen. Im Schnittpunkt der Ordnungslinie darch $a^{\prime}$ mit der Parallelen durch den Punkt $a^{s \prime \prime}$ zu $L^{\prime \prime}$ liegt $a^{\prime \prime}$. $a^{\prime \prime}$ kann auch erhalten werden, indem man die Strecke $\overline{12}=d=\overline{a^{8} a^{\prime}}$ macht und durch den Punkt 2 unter dem Winkel $\alpha$ gegen den Horizont eine Gerade zieht, wenn $\alpha$ den Neigungswinkel der Tangenten von $S$ gegen die Horizontebene bedeutet. Der Aufriß kann also hier viel einfacher ermittelt werden als bei beliebigen Schraubflächen. Der Schnittpunkt $t_{a}$ der Tangente aus $o^{0}$ an $\overline{S^{s}}$ mit dem Horizont $H$ ist bereit ein Punkt der Konturtangente in $a^{c}$.

Durch jeden Punkt $p$ von $S$ geht eine Schraublinie $\bar{S}$. Projizieren wir diese Schraublinie $\bar{S}$ in der Richtung der Tangente in $p$ an $S$ auf die Horizontebene, so erscheint jeder durch ein Linienelement von $S$ gegebenen infinitesimalen Schiebnng eine bestimmte Kurve $\bar{S}^{s}$ zugeordnet. Diese Kurve $\bar{S}^{s}$ soll „der der infinitesimalen Schiebung zugeordnete Sehrägrib" heißen. Nun sind alle Schraublinien $\bar{S}$ kongruent und gleichgestellt und andererseits die sämtlichen durch die Tangenten von $S$ bestimmten

8) Man vgl. L. Tuschel: Über eine krummlinige Projektion and deren Verwendung in der darstellenden Geometrie, Monatshefte f. Math. u. Phys. Bd. 20, pag. 366 .

$\left.{ }^{9}\right)$ In Fig. 4 ist nur eine Tangente gezeichnet. 
infinitesimalen Verschiebungsrichtungen gleich geneigt gegen die Horizontebene, Daher gilt:

Die den sämtlichen infinitesimalen Schiebungen zugeordneten Schrägrisse sind kongruent.

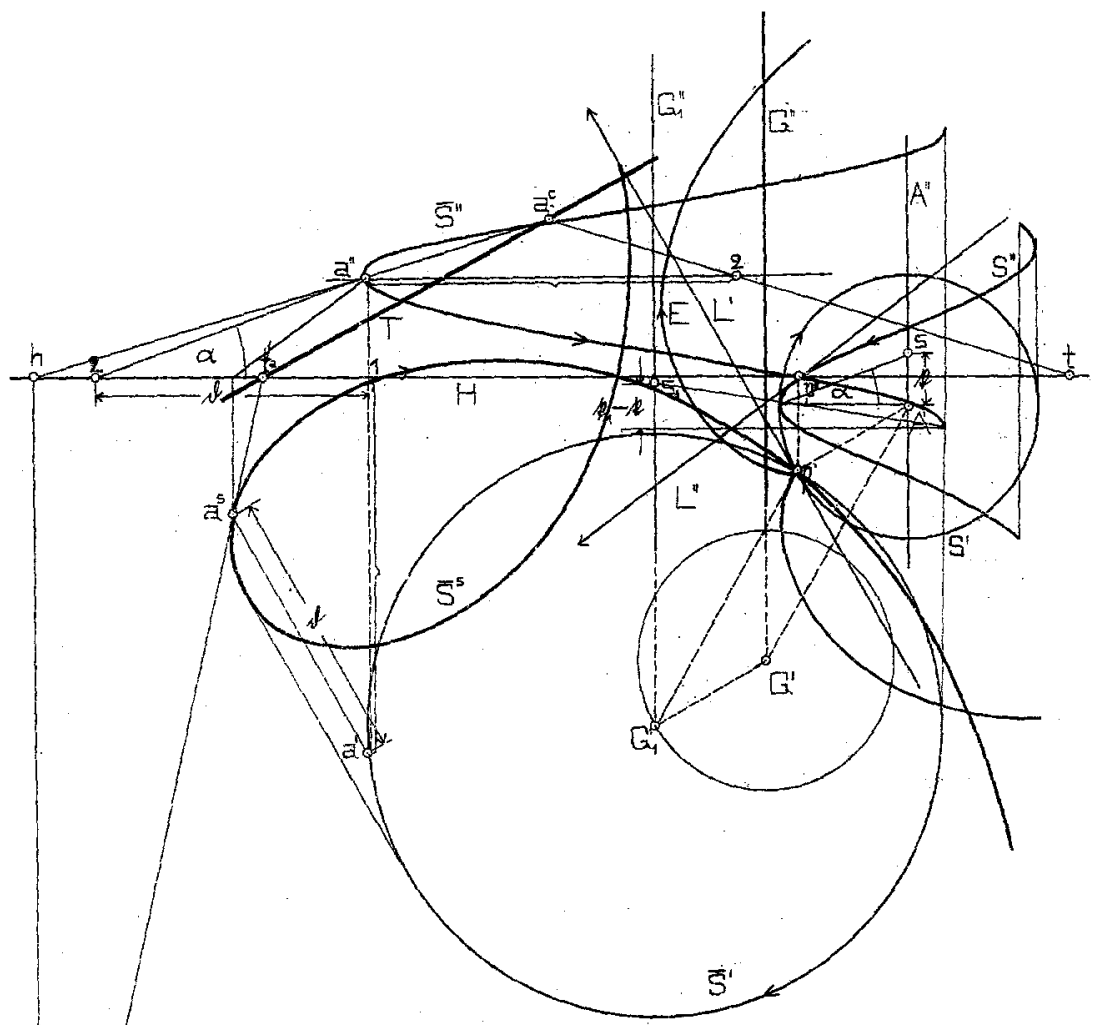

Fig. 4 .

Man wird daher bei der praktischen Durchführung der Konturbestimmung das Pauspapier verwenden. Wir verzeichnen mithin $\bar{S}^{s}$ und $L^{\prime}$ auf einem Pauspapier. Es handelt sich jetzt nur darum, wie wir dasselbe in die Zeichenebene hineinlegen. Unterwerfen wir $\bar{S}$ der krummen Sehiebung längs $S$, so beschreibt der Schrägriß des auf $S$ sich bewegenden Punktes $p$ von $\bar{S}$ in der Horizont$0_{0^{\circ}}$ ebene die gemeine Kreisevolvente $E$ des Kreises $S^{\prime}$ für
den Punkt $p$. Der Grundrib $G_{1}^{\prime}$ der Achse von $\bar{S}$ beschreibt hiebei einen Kreis mit dem Mittelpunkte $G^{\prime}$ und dem Radius $\overline{G^{\prime} G_{1}^{\prime}}=\overline{A^{\prime} p}$. Die Lage des Pauspapiers ist also dadurch gegeben, dab $p^{s}$ sich auf $E$ befindet und $L^{\prime}$ orientierte Tangente an den orientierten 
Kreis $S^{\prime}$ ist. Die sich ergebenden Punkte $a^{s}$ und $t_{\alpha}$ drückt man mit einem Pausstift durch. Aus $a^{s}$ ergibt sich $a^{\prime}$ auf dem durch den Berührungspunkt von $L^{\prime}$ mit $S^{\prime}$ gehenden orientierten Kreis $\bar{S}^{\prime}$. $a^{\prime \prime}$ findet man durch Abtragen der Strecke $\overline{a^{s} a^{\prime}}$ auf dem Horizont $H$ und Ziehen einer gegen $\hat{H}$ unter dem Winkel $\alpha$ geneigten Geraden. Hat man also die Zykloide $\bar{S}^{s}$ und die Erolvente $E$ gezeichnet, so läbt sich die ganze Kontur mit Hilfe des Pauspapiers sehr einfach und vollkommen streng konstruieren.

Diese Methode der Konstruktion des perspektiven Umrisses ist bei allen Schiebflächen, dereneine Erzeugende eine Böschungslinie ist, anwendbar.

Zum Schlusse sei noch bemerkt, daß man in derselben einfachen Weise den perspektiven Umriß jener Hüllschraubflächen konstruieren kann, die durch Schraubung einer Schraubfläche $\varphi$ um eine zu ihrer Achse $A$ parallele Schraubacbse $G$ ents tehen. Diese Hüllschraubflächen können, wie E. Müller ${ }^{10}$ ) gezeigt hat, auch durch krumme Schiebung erzeugt werden. Zur praktischen Durchführung der Konturbestimmung wird man daher für eine Fläche $\varphi$ die Eigenschattengrenze und den Schlagschatten auf die Horizontebene für die Richtung jener infinitesimalen Schiebung, die die Fläche $\varphi$ in die Nachbarlage überführt, als Lichtrichtung ermitteln und zur weiteren Konstruktion das Pauspapier verwenden.

10) E. Müller: Über die Hülleächen von Flächenscharen, die durch krumme Schiebung erzengt werden. Monatshefte f. Math. u. Phys. Bd. 21, pag. $150-154$. 